LA W RENCE LIVERMORE N A T IO N A L LABORATORY

\title{
Optical properties of passivated silicon nanoclusters: the role of synthesis
}

E.W. Draeger, J.C. Grossman, A.J. Williamson, G.G. Galli

April 16, 2004

Journal of Chemical Physics 
This document was prepared as an account of work sponsored by an agency of the United States Government. Neither the United States Government nor the University of California nor any of their employees, makes any warranty, express or implied, or assumes any legal liability or responsibility for the accuracy, completeness, or usefulness of any information, apparatus, product, or process disclosed, or represents that its use would not infringe privately owned rights. Reference herein to any specific commercial product, process, or service by trade name, trademark, manufacturer, or otherwise, does not necessarily constitute or imply its endorsement, recommendation, or favoring by the United States Government or the University of California. The views and opinions of authors expressed herein do not necessarily state or reflect those of the United States Government or the University of California, and shall not be used for advertising or product endorsement purposes. 


\title{
Optical properties of passivated silicon nanoclusters: the role of synthesis
}

\author{
Erik W. Draeger, Jeffrey C. Grossman, Andrew J. Williamson, and Giulia Galli \\ Lawrence Livermore National Laboratory, Livermore, CA 94550
}

\begin{abstract}
The effect of preparation conditions on the structural and optical properties of silicon nanoparticles is investigated. Nanoscale reconstructions, unique to curved nanosurfaces, are presented for silicon nanocrystals and shown to have lower energy and larger optical gaps than bulk-derived structures. We find that high-temperature synthesis processes can produce metastable noncrystalline nanostructures with different core structures than bulk-derived crystalline clusters. The type of core structure that forms from a given synthesis process may depend on the passivation mechanism and time scale. The effect of oxygen on the optical of different types of silicon structures is calculated. In contrast to the behavior of bulk-like nanostructures, for noncrystalline and reconstructed crystalline structures surface oxygen atoms do not decrease the gap. In some cases, the presence of oxygen atoms at the nanocluster surface can significantly increase the optical absorption gap, due to decreased angular distortion of the silicon bonds. The relationship between strain and the optical gap in silicon nanoclusters is discussed.
\end{abstract}

\section{INTRODUCTION}

In recent years, there has been a considerable amount of theoretical and experimental interest focused on silicon nanoclusters [1-4]. Silicon quantum dots between 1-3 nm in 
diameter can exhibit luminescence at visible wavelengths as the optical absorption gap is enhanced by quantum confinement effects. By controlling the size of silicon clusters one can precisely tune the color of the resulting flourescence. This fact, coupled with their compatibility with existing silicon-based technologies, make silicon quantum dots very promising for numerous technological applications.

One of the fundamental challenges to the theoretical investigation of nanoclusters is the choice of atomic configurations. The most common type of silicon nanostructure to be investigated theoretically is a spherical piece of bulk crystalline material, usually with the surface ideally terminated with hydrogen atoms to saturate dangling bonds. Recent theoretical work has shown that highly curved spheres of bulk crystalline material may reconstruct, requiring less hydrogen to fully passivate the surface than in the ideally terminated geometry [7]. In addition, ab initio calculations have pointed out surface reconstructions that are unique to nanocrystals, which are different from those of bulk solids [8]. We show that for the same stoichiometry there exist several reconstructions which arise from multiple ways to connect a nanocrystal core to a curved surface [9]. We also use first-principles molecular dynamics to examine the types of non-crystalline $1 \mathrm{~nm}$ nanostructures that arise from high temperature synthesis and compare their optical properties with those of crystalline structures of the same size.

Three different types of $1 \mathrm{~nm}$ silicon nanostructures are compared: unreconstructed crystalline, reconstructed crystalline, and "reconstructed" non-crystalline. These non-crystalline structures are generated using first-principles molecular dynamics and in general they differ from non-crystalline nanoclusters created from bulk amorphous templates used in previous theoretical studies [5].

The experimentally determined optical properties of silicon quantum dots can vary significantly depending on the synthesis technique and preparation conditions $[11,10,12-17]$. Our results shows that the likelihood of a given type of structure forming strongly depends on the synthesis conditions. A rigorous investigation of different types of nanocluster structures in a given size range may be necessary to make general theoretical predictions of nanoclus- 
ters obtained under different synthesis conditions. To illustrate this point, we investigate how the optical absorption gap of $1 \mathrm{~nm}$ silicon nanoclusters varies with reconstruction, the presence of surface oxygen, and hydrostatic strain for different types of nanostructures. These calculations can help explain current experimental results, and may provide an optical "fingerprint" for different nanostructures. The systematic comparison of different types of structures (reconstructed vs. unreconstructed, crystalline vs. non-crystalline) presented here is not limited to silicon nanoclusters, but can be generalized to a variety of curved nanoscale systems.

\section{METHOD}

A variety of first-principles methods were used to investigate the effect of synthesis conditions on the optical properties of $1 \mathrm{~nm}$ nanoclusters. Density functional theory (DFT) was used for structural relaxation, as well as calculating trends in the total energy and the optical absorption gap. First-principles molecular dynamics (FPMD) was used to simulate the dynamics of nanostructure formation and amorphization, with a time-step of 0.075 fs. Both DFT and FPMD calculations were performed using the GP 1.16.0 plane wave code [18], with the local density approximation (LDA), and Hamann [19] (silicon) and Giannozzi [20] (hydrogen) pseudopotentials. A 12 Ry cutoff was used for FPMD, while for the static DFT calculations a larger 35 Ry cutoff was used to obtain accurate energy differences and fully converged unoccupied orbitals. Quantum Monte Carlo (QMC) was used to calculate total energy differences and optical absorption gaps which can be directly compared to experiment. The CASINO QMC code was used [21] with the fixed-node diffusion Monte Carlo (DMC) implementation of QMC, in the same manner as in Ref. [22]. Unless otherwise noted,

all energy differences and optical absorption gaps reported here were calculated using QMC. 


\section{CURVED NANOSURFACE RECONSTRUCTIONS}

The most straightforward choice of nanocluster structures for a given number of silicon atoms is a spherical piece of bulk with all dangling bonds passivated with hydrogen [23-27]. However, it has been shown that crystalline cluster surfaces can reconstruct and in particular, because of the high curvature of nanoscale clusters, there can be multiple ways to connect the core to the surface. It is important to take the curvature of the surface into account when making theoretical predictions.

For $1 \mathrm{~nm}$ silicon clusters, a bulk-like surface dimerization yields $\mathrm{Si}_{29} \mathrm{H}_{24}^{b u l k}$, instead of the ideally-terminated $\mathrm{Si}_{29} \mathrm{H}_{36}$, as shown in Fig. 1. Taking the highly curved surface into account, we find that $\mathrm{Si}_{29} \mathrm{H}_{24}$ has two additional surface reconstructions $\left(\mathrm{Si}_{29} \mathrm{H}_{24}^{\text {nano1 }}\right.$ and $\mathrm{Si}_{29} \mathrm{H}_{24}^{\text {nano2 }}$ ) generated by reconnecting the core to different surface atoms. The $\mathrm{Si}_{29} \mathrm{H}_{24}^{\text {nano1 }}$ structure differs from $\mathrm{Si}_{29} \mathrm{H}_{24}^{\text {bulk }}$ only by the fact that the interior core atom is bonded to four different atoms at the surface of the cluster, with hydrogen atoms moved appropriately so that all silicon atoms are four-fold coordinated and there are no dangling bonds. A single such bond transfer is illustrated in the inset of Fig. 1. The atoms that bond to the interior core atom in $\mathrm{Si}_{29} \mathrm{H}_{24}^{\text {nano1 }}$ are neighbors of the atoms that bond to the interior core atom in $\mathrm{Si}_{29} \mathrm{H}_{24}^{\text {bulk }}$, chosen so that the five-atom core still retains tetrahedral symmetry. The $\mathrm{Si}_{29} \mathrm{H}_{24}^{\text {nano2 }}$ structure involves the change of only three silicon-silicon bonds from $\mathrm{Si}_{29} \mathrm{H}_{24}^{b u l k}$. The three atoms that now bond to the core are next-nearest neighbors of those in $\mathrm{Si}_{29} \mathrm{H}_{24}^{b u l k}$, chosen to maintain a tetrahedrally symmetric core. Alternatively, one can think of these clusters as a 28-atom fullerene cage surrounding a single core atom - these reconstructions represent the three unique ways to connect the fullerene and produce a tetrahedrally-symmetric core.

It should be noted that although we have described the new structures using $\mathrm{Si}_{29} \mathrm{H}_{24}^{\text {bulk }}$ for reference, we are not asserting that these structures necessarily form from this starting point. Depending on the synthesis conditions, different structures may initially form which are kinetically but not necessarily thermodynamically limited.

We find that both the $\mathrm{Si}_{29} \mathrm{H}_{24}^{\text {nano1 }}$ and $\mathrm{Si}_{29} \mathrm{H}_{24}^{\text {nano2 }}$ reconstructions are lower in energy than 
the bulk-derived $\mathrm{Si}_{29} \mathrm{H}_{24}^{\text {bulk }}$ structure. The DMC energies of $\mathrm{Si}_{29} \mathrm{H}_{24}^{\text {nano1 }}$ and $\mathrm{Si}_{29} \mathrm{H}_{24}^{\text {nano2 }}$ are 0.6(1) eV and 1.0(1) eV lower than $\mathrm{Si}_{29} \mathrm{H}_{24}^{\text {bulk }}$, respectively, due to decreased strain around the surface dimers. Analogous structures were created from the unreconstructed bulk fragment $\mathrm{Si}_{29} \mathrm{H}_{36}$ and found to be higher in energy. This supports the idea that these reconstructions are energetically favored in structures with a reconstructed surface as a result of lower strain in the silicon-silicon dimers at the curved surface, which are absent in unreconstructed $\mathrm{Si}_{29} \mathrm{H}_{36}$. In addition, a partial reconstruction from $\mathrm{Si}_{29} \mathrm{H}_{24}^{\text {bulk }}$ to $\mathrm{Si}_{29} \mathrm{H}_{24}^{\text {nano1 }}$, in which a single surface core atom takes the hydrogen from its neighbor and bonds to the central core atom (see Fig. 1 inset), also has lower energy than $\mathrm{Si}_{29} \mathrm{H}_{24}^{\text {bulk }}$. Therefore, reconstruction from $\mathrm{Si}_{29} \mathrm{H}_{24}^{\text {bulk }}$ to $\mathrm{Si}_{29} \mathrm{H}_{24}^{\text {nano1 }}$ only involves the breaking of a single silicon bond at a time.

The reconstructions discussed here are not unique to $1 \mathrm{~nm}$ clusters. For example, $\mathrm{Si}_{66} \mathrm{H}_{40}$

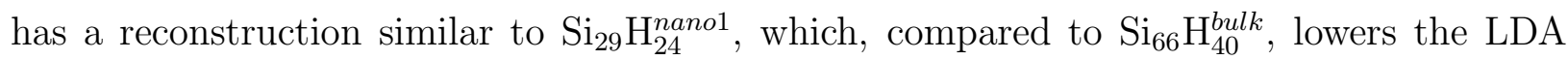
total energy by $0.46 \mathrm{eV}$, and increases the DFT highest occupied molecular orbital-lowest unoccupied molecular orbital (HOMO-LUMO) gap from $2.02 \mathrm{eV}$ to $2.22 \mathrm{eV}$. In general, our results suggest that exploring different ways of connecting a nanocluster core to a curved surface is a necessary component of a thorough theoretical investigation of a nanoscale system.

\section{HIGH TEMPERATURE SYNTHESIS}

Several experimental techniques for synthesizing silicon nanoparticles, such as physical vapor deposition [12] and laser ablation [28], generate a high temperature silicon vapor from which nanoclusters nucleate as the vapor cools. During or after cooling, there is typically exposure to some form of passivant (e.g., hydrogen, oxygen) which removes dangling bond states from the surface of the cluster. To simulate this process, we start from an amorphous cluster obtained by heating a bare silicon cluster (with ideal crystalline geometry) at $1500 \mathrm{~K}$ for $\sim 3$ ps to remove any "memory" of the initial conditions. A new hydrogen atom is added to the surface every $0.45-0.75$ ps until all silicon atoms are four-fold coordinated. 
Several different algorithms for attaching the hydrogens to available surface silicon atoms were tested. Most of the simulations performed used an algorithm in which a new hydrogen atom is placed on the available (i.e. a silicon atom with less than four neighbors) surface silicon which is farthest from any other hydrogen, so as to maximize the overall uniformity of hydrogen distribution on the surface. Other rules we employed to choose between multiple available passivation sites included choosing surface silicon atoms with the maximum number of dangling bonds, maximizing the new hydrogen distance from the cluster center of mass and/or simple random sampling.

We found that as long as hydrogen atoms were attached to silicon atoms with a coordination of less than four, the properties such as HOMO-LUMO gap and atomization energy were fairly independent of the algorithm (and hence the order) by which hydrogen atoms were attached to the surface. Simple thermodynamic arguments, assuming atmospheric pressure and ideal gas vapor densities, predict an additional hydrogen will passivate the surface every 1-2 ps. By using a series of elevated temperatures ( $\mathrm{T}=600,800$, and $1000 \mathrm{~K})$, we were able to model realistic passivation events on shorter time scales. For each system, multiple simulations were carried out from different starting points in order to obtain a statistically representative sample. The combined total simulation time for all clusters investigated here was $\sim 750$ ps.

The present work focuses on the $1 \mathrm{~nm}$ size range where there are $\sim 30$ silicon atoms per quantum dot. We performed simulations starting from amorphous $\mathrm{Si}_{29}$ and $\mathrm{Si}_{30}$. Surprisingly, in simulations started from $\mathrm{Si}_{29}$, the single tetrahedral core structure of 29 silicon atoms (see Fig. 1) does not form. Instead, we found that the resulting passivated structures consistently had a double tetrahedral core - two interior silicon atoms rather than one (see Fig. 2). The formation of a double core was found to be independent of temperature and the order in which hydrogen atoms were added to the surface. Eight separate synthesis calculations starting from amorphous $\mathrm{Si}_{29}$ all resulted in double-core structures. The resulting $\mathrm{Si}_{29}$ structures were highly strained due to the lack of a symmetric arrangement of 21 surface silicon atoms around the 8-atom double core geometry. 
The explanation for the consistent formation of a double-core structure lies with the dynamics of bare silicon clusters. In our simulations above $\mathrm{T}=300 \mathrm{~K}$, unpassivated crystalline nanoclusters become amorphous with multiple atoms in the center of the cluster attempting to saturate the dangling bonds of the surface atoms. $1 \mathrm{~nm}$ clusters of amorphous silicon typically have two to three such highly coordinated interior atoms. As hydrogens are added to the surface, the number of dangling bonds is reduced and the interior atoms develop stable bonds to the surface. As long as a majority of the atoms at the surface are unpassivated, it is energetically favorable for two interior atoms to each passivate three dangling bonds to form an eight atom double tetrahedral core. Passivation has the effect of "freezing" that region of the cluster surface as atoms at the surface become four-fold coordinated. By the time enough hydrogens have been added to fully passivate the surface, the cluster surface has frozen around a double-core structure. During formation, there is no clear pathway to a single-core structure in this type of synthesis process, as forming a single-core cluster from bare silicon requires a portion of the surface to sustain multiple dangling bonds until enough hydrogen atoms have been added to fully passivate the surface; this situation is likely to occur only when starting from a bare crystalline cluster at low temperature and using rapid passivation. Once a double-core cluster is formed, a transition to a single-core structure would involve a global reconstruction of the surface, with multiple events involving the simultaneous breaking of several silicon bonds, and thus is expected to have a low probability to occur.

By symmetry, we may expect 30 silicon atoms to be energetically more favorable than 29 atoms for the formation of $1 \mathrm{~nm}$ double-core nanoclusters. Six subsequent simulations of hydrogen passivation of amorphous $\mathrm{Si}_{30}$ at $\mathrm{T}=600 \mathrm{~K}$ and $\mathrm{T}=1000 \mathrm{~K}$, using the same FPMD synthesis calculations as for $\mathrm{Si}_{29}$, again consistently produced double-core structures The simulation dynamics of $\mathrm{Si}_{29}$ and $\mathrm{Si}_{30}$ were qualitatively very similar. However, FPMD simulations starting from $\mathrm{Si}_{30}$ produced several low-energy structures (the example shown in Fig. 3 had a total energy which is only $0.1 \mathrm{eV}$ higher than the ideal structure $\mathrm{Si}_{30} \mathrm{H}_{22}^{\text {ideal }}$ ) that are qualitatively similar to the ideal high-symmetry structure. $\mathrm{Si}_{30} \mathrm{H}_{22}$ has a symmetric 
ideal construction which can be represented as a 28-atom cage (14 pentagons, 2 septagons) surrounding two interior atoms (Fig. 3). To test stability, we heated this structure at $\mathrm{T}=1000 \mathrm{~K}$ for $15 \mathrm{ps}$, and found it to be stable to thermodynamic fluctuations. Fig. 3 also shows two curved nanosurface reconstructions of $\mathrm{Si}_{30} \mathrm{H}_{22}$. Although both of these are higher in energy than the proposed "ideal" structure, they are included here as additional examples of curved surface reconstructions.

To study how the core structure evolves as the synthesis simulation progresses, one needs a quantitative measure of whether a given silicon atom is in the interior of the cluster. We define the degree to which an atom is in the interior by the fraction of the total solid angle around atom $i$ that is not occupied by other silicon atoms. For simplicity, we approximate this solid angle by calculating the maximum base angle $\Omega$ of a cone whose apex is centered on atom $i$ and whose volume does not intersect any other silicon atoms (see Fig. 4 inset):

$$
\begin{aligned}
\Omega_{i}= & \max \left\{\theta_{1}, \cdots, \theta_{i-1}, \theta_{i+1}, \cdots, \theta_{N}\right\} \\
& -\min \left\{\theta_{1}, \cdots, \theta_{i-1}, \theta_{i+1}, \cdots, \theta_{N}\right\}
\end{aligned}
$$

where $0 \leq \theta_{j}<2 \pi$ is the angle atom $j$ makes with the axis defined by the vector from the center of mass to atom $i$, translated so that atom $i$ is at the origin (the center of mass is at $\theta=\pi)$. An atom in the center of an infinite solid will have a value of $\Omega$ which approaches zero. For a small nanocluster, the minimum value of $\Omega$ for an interior atom is roughly 30 degrees, due to the finite number of atoms. An atom at the surface will typically have a value of $120<\Omega<230$ degrees, depending on whether it is a core (and hence directly bonded to an interior atom) or a surface atom passivated with hydrogen.

The quantity $\Omega$ is plotted as a function of time in Fig. 4 for a simulation of the synthesis of $\mathrm{Si}_{30}$, starting from an amorphous silicon cluster. To create the amorphous silicon cluster, a bare crystalline $\mathrm{Si}_{29}$ cluster was heated at $\mathrm{T}=1500 \mathrm{~K}$ for 3 ps to remove all "memory" of the initial conditions. This was used as the starting point for $\mathrm{Si}_{29}$ FPMD runs. For $\mathrm{Si}_{30}$ FPMD simulations, an additional silicon atom was added to the surface of an amorphous $\mathrm{Si}_{29}$ cluster and heated at $\mathrm{T}=1000 \mathrm{~K}$ for an additional $1.5 \mathrm{ps}$ before any hydrogen atoms 
were added. In the early stages of the synthesis process, before many hydrogen atoms have been added to the surface, fluctations in $\Omega$ are large, and exchange of interior and surface atoms is common. Once roughly half of the surface dangling bonds are passivated, the fluctuations in $\Omega$ of the interior atoms decrease considerably and exchange between the core and the surface was never observed. These results suggest that $\Omega$ can be used to gauge the impact of the surface of a nanostructure on the dynamics of the core structure.

\section{CALCULATIONS OF OPTICAL ABSORPTION GAPS}

Within DFT, the optical absorption gap is defined as the energy required to excite an electron from the highest occupied molecular orbital (HOMO) into the lowest unoccupied molecular orbital (LUMO). Although it is well-known that the DFT HOMO-LUMO gap is consistently lower than the measured optical absorption gap, previous studies of crystalline silicon nanoclusters have shown that DFT accurately reproduces optical gap trends as a function of size $[25,22]$. On the other hand, optical absorption gaps calculated with QMC using relaxed DFT ground-state structures are expected to be directly comparable to experimental absorption measurements.

Table I shows the optical absorption gaps calculated with both DFT and QMC for the three different types of structures considered in this paper. The unreconstructed crystalline $\mathrm{Si}_{29} \mathrm{H}_{36}$ cluster has the largest optical absorption gap of 5.3(1) eV. Reconstruction to crystalline $\mathrm{Si}_{29} \mathrm{H}_{24}$ lowers the optical absorption gap by $0.8-1.8 \mathrm{eV}$, depending on the reconstructed structure. A range of DFT gaps is provided to give an estimate of the statistical variation of our FPMD results. The three non-crystalline QMC optical absorption gaps were all 1.0(1) eV larger than the LDA values. If one assumes the LDA-QMC offset is also $1 \mathrm{eV}$ for the other non-crystalline $1 \mathrm{~nm}$ clusters, these double-core structures would consistently have optical absorption gaps between 3.2-3.4 eV.

The most striking feature of Table I is the large variation in the optical absorption gaps

of the $1 \mathrm{~nm}$ crystalline structures. Even among the reconstructed $\mathrm{Si}_{29} \mathrm{H}_{24}$ structures, which 
have the same stoichiometry, the QMC optical absorption gaps vary by $1 \mathrm{eV}$. The total energy of the three structures also varies by $1 \mathrm{eV}$. Although the $\mathrm{Si}_{29} \mathrm{H}_{24}^{\text {bulk }}$ structure has both the highest energy and lowest optical absorption gap, the inverse is not true: the lowest en-

ergy structure, $\mathrm{Si}_{29} \mathrm{H}_{24}^{\text {nano2 }}$, does not have the largest optical absorption gap. In contrast, the non-crystalline double core structures produced by high temperature FPMD synthesis calculations have optical gaps which are remarkably insensitive to strain. The calculated optical absorption gaps of relaxed FPMD final structures are consistently within $\sim 0.1-0.2 \mathrm{eV}$, even though the total energies vary by $\sim 2-3 \mathrm{eV}$. This insensitivity of the gap to energy suggests that, in the absence of defect states introduced by e.g. chemical contaminants, passivated $1 \mathrm{~nm}$ silicon nanostructures have a minimum optical absorption gap determined by quantum confinement. The reason for the increased optical absorption gap in the crystalline clusters is likely a combination of symmetry and reduced angular distortion in the silicon-silicon bonds. This will be discussed in more detail later in Section VII.

It should be noted that previous theoretical studies [5] using empirical tight binding (ETB) calculations found that both bare and hydrogenated amorphous $1 \mathrm{~nm}$ silicon clusters have optical absorption gaps of over $3 \mathrm{eV}$, similar to the QMC gaps of non-crystalline structures produced by FPMD synthesis shown in Table I. However, while the ETB studies found that the presence of hydrogen has only a small effect on the optical absorption gap, our ab initio calculations find that full passivation of the surface is essential to obtain large optical absorption gaps in these systems. In fact, we found that even a single dangling bond state will typically reduce the LDA optical absorption gap by more than $1 \mathrm{eV}$.

\section{EFFECT OF SURFACE OXYGEN}

A number of experimental synthesis techniques result in silicon quantum dot surfaces that are exposed to oxygen either during or after the synthesis process. Therefore, understanding the effect of surface contaminants such as oxygen on the optical absorption gap of silicon nanoclusters can help interpret experimental data. Previous studies [30,26,31,32,29,27] found 
that in certain cases the presence of oxygen can significantly lower the optical gap of unreconstructed silicon dots with a crystalline core. Our results confirm these earlier studies; in addition, we show that the effect of oxygen on the surface of reconstructed or non-crystalline silicon nanoparticles can be quite different than for unreconstructed (i.e., bulk surface) crystalline ones. For example, oxygen passivation of crystalline silicon quantum dots with a reconstructed surface may not lower the gap and instead could actually increase the gap. Furthermore, for non-crystalline (i.e., amorphous) structures we find that oxygen has a negligible impact on the optical absorption gap. These results suggest that synthesis techniques that are likely to produce reconstructed or non-crystalline nanostructures yield clusters with optical absorption gaps that are robust to the presence of oxygen, making these techniques more attractive for certain applications. In fact, the variation of optical absorption gaps with oxygen may itself be used as an experimental measure of nanocluster structure.

The effect of oxygen on unreconstructed silicon nanocrystals has been investigated [32,29] for a range of cluster sizes. For the $1 \mathrm{~nm}$ case, the addition of a single oxygen atom to the surface of $\mathrm{Si}_{29} \mathrm{H}_{36}$ (replacing two hydrogen atoms to form $\mathrm{Si}_{29} \mathrm{H}_{34} \mathrm{O}$ ) lowers the QMC optical absorption gap by $0.6 \mathrm{eV}$ when attached in a bridged configuration ( $\mathrm{Si}-\mathrm{O}-\mathrm{Si}$ ), and by $2.8 \mathrm{eV}$ when attached in a double-bonded configuration $(\mathrm{Si}=\mathrm{O})$. The LDA total energy of $\mathrm{Si}_{29} \mathrm{H}_{34} \mathrm{O}$ with a double-bonded oxygen is $2.2 \mathrm{eV}$ higher than the same cluster with a bridged oxygen, and thus the double-bonded configuration is unlikely to occur for this stoichiometry and size. In general, for unreconstructed silicon nanoclusters, the bridged configuration was found to be lower in energy for surfaces where silicon-silicon dimers were present [26].

For the reconstructed $\mathrm{Si}_{29} \mathrm{H}_{24}$, we find that attaching oxygen atoms in a bridged configuration has a dramatically different effect on the optical absorption gap depending on

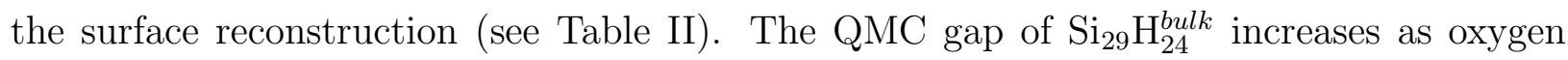
atoms are added to the surface, the gap of $\mathrm{Si}_{29} \mathrm{H}_{24}^{\text {nano1 }}$ significantly decreases, and the gap of $\mathrm{Si}_{29} \mathrm{H}_{24}^{\text {nano2 }}$ remains constant within statistical error bars. It should be noted that although all surface dimers of $\mathrm{Si}_{29} \mathrm{H}_{24}^{\text {bulk }}$ are equivalent by symmetry, both $\mathrm{Si}_{29} \mathrm{H}_{24}^{\text {nano1 }}$ and $\mathrm{Si}_{29} \mathrm{H}_{24}^{\text {nano2 }}$ have multiple sites for attaching a single bridged oxygen atom, only one of which was cal- 
culated here. We expect the variation in the gap due to these multiple sites to be smaller than the difference in the gap between the single oxygen case and the fully oxygenated case. Adding an oxygen atom to the FPMD generated structures shown in Fig. 3 does not change the optical absorption gap by a measurable amount. The effect of oxygen on the HOMO and LUMO states of each reconstruction is shown in Fig. 5. The nature of the HOMO and LUMO states is consistent with the observed changes in the optical absorption gap. For

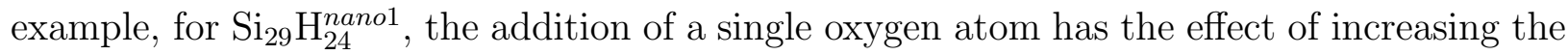
localization of the HOMO, thereby raising its energy and decreasing the HOMO-LUMO gap. The addition of six oxygen atoms further delocalizes the LUMO, causing a corresponding decrease in the energy of the state, and decrease of the gap. One common feature to all three reconstructions is the fact that neither the HOMO nor LUMO state is significantly localized on the oxygen atom(s).

In addition to changing the optical absorption gap, surface oxygen atoms change the relative energetics of the three $\mathrm{Si}_{29} \mathrm{H}_{24}$ reconstructions. Without oxygen, the $\mathrm{Si}_{29} \mathrm{H}_{24}^{\text {nano2 }}$ reconstruction is the lowest in total DMC energy, 0.4 and $1.0 \mathrm{eV}$ lower than $\mathrm{Si}_{29} \mathrm{H}_{24}^{\text {nano1 }}$ and $\mathrm{Si}_{29} \mathrm{H}_{24}^{\text {bulk }}$, respectively. When a single oxygen atom is added, the $\mathrm{Si}_{29} \mathrm{H}_{24}^{\text {nano2 }}$ reconstruction is the highest energy structure, 1.8 and $0.6 \mathrm{eV}$ higher than $\mathrm{Si}_{29} \mathrm{H}_{24} \mathrm{O}^{\text {nano1 }}$ and $\mathrm{Si}_{29} \mathrm{H}_{24} \mathrm{O}^{\text {bulk }}$, respectively. In the case of full oxygenation of the surface, whereby 6 oxygen atoms are attached, $\mathrm{Si}_{29} \mathrm{H}_{24} \mathrm{O}_{6}^{\text {nano2 }}$ is again the lowest energy reconstruction, 0.4 and $0.9 \mathrm{eV}$ lower than $\mathrm{Si}_{29} \mathrm{H}_{24} \mathrm{O}_{6}^{\text {bulk }}$ and $\mathrm{Si}_{29} \mathrm{H}_{24} \mathrm{O}_{6}^{\text {nano1 }}$, respectively.

Previous theoretical calculations $[26,31,32,29,27]$ of unreconstructed crystalline nanoclusters found that the optical absorption gap was consistently and significantly reduced by the addition of oxygen atoms to the surface. Puzder et al. found the gap reduction was much larger if the oxygen atom was double-bonded to the cluster due to a localization of the HOMO and LUMO states. The calculations presented here show a different picture for the the non-crystalline cases; namely, the effect of adding surface oxygen atoms to nanoclusters is purely structural, and thus can either decrease or increase the optical absorption gap. For experimental techniques which allow precise control over the presence of oxygen during 
synthesis, the relationship between gaps and structure presented here may provide an indirect probe of the cluster structure, by comparing the absorption gaps of clusters synthesized with and without oxygen with theoretical calculations.

\section{EFFECT OF STRAIN}

The variation of gap values with surface geometry found here and the fact that oxygen atoms can actually increase the gap of $\mathrm{Si}_{29} \mathrm{H}_{24}^{b u l k}$ show that oxygen influences silicon-silicon bonds. Moreover, angular distortion is known to significantly impact the absorption gaps of silicon systems. For example, the gap of $\mathrm{SiH}_{4}$ has been shown to change significantly when the $\mathrm{H}-\mathrm{Si}-\mathrm{H}$ bond angle is distorted [32], with an increased strain of less than $0.4 \mathrm{eV}$ corresponding to a reduction in the LDA HOMO-LUMO gap of over $1 \mathrm{eV}$. In contrast, the gap of $\mathrm{SiH}_{4}$ changes by only $0.3 \mathrm{eV}$ when $0.8 \mathrm{eV}$ of strain is applied by uniformly compressing the Si-H bonds. For the clusters considered in this work, we analyzed the impact of oxygen on reconstructed crystalline and non-crystalline structures by comparing the relative distortion of the silicon bond angles caused by the oxygen in different geometries. In fact, the effect of bridged oxygen on the unreconstructed $\mathrm{Si}_{29} \mathrm{H}_{36}$ cluster is very similar to that of the surface dimers formed by reconstruction to $\mathrm{Si}_{29} \mathrm{H}_{24}$, i.e. the bond angles of the silicon atoms involved in the double bond/surface dimer are distorted and the optical absorption gap decreased.

In an attempt to quantify the effect of angular distortion on the optical gap, Fig. 6 shows the QMC optical absorption gap as a function of the root-mean-square deviation of the SiSi-Si bond angles from the bulk silicon bond angle, for all silicon nanostructures investigated in this paper. Although the plot does not take into account the different stoichiometries and symmetries of the clusters, there appears to be a general relationship between decreased angular distortion and increased optical absorption gap. However, this does not itself prove that angular distortion is responsible for decreasing the optical absorption gap, as clusters with small angular distortion also tend to have lower total strain energy.

In order to introduce strain without changing the bond angles or cluster symmetry, the 
unreconstructed $\mathrm{Si}_{29} \mathrm{H}_{36}$ cluster and the three reconstructions of $\mathrm{Si}_{29} \mathrm{H}_{24}$ were compressed uniformly by a simple coordinate rescaling. The optical absorption gap of the resulting clusters as a function of total energy is plotted in Fig. 7. It is striking that the optical absorption gap is not only insensitive to hydrostatic compression, but initially increases

for both $\mathrm{Si}_{29} \mathrm{H}_{24}^{\text {bulk }}$ and $\mathrm{Si}_{29} \mathrm{H}_{24}^{\text {nano2 }}$, corresponding to a negative deformation potential of the optical absorption gap. This suggests that the optical absorption gap does not depend on the amount of strain energy per se, but on combinations of angular distortion and symmetry.

Clusters with no discernable symmetry (e.g. those produced by FPMD) appear to have some minimum optical absorption gap based on quantum confinement and surface chemistry. The proposed lower bound for $1 \mathrm{~nm}$ silicon nanoclusters without dangling bond states or localized impurity states (e.g. double-bonded oxygen) is estimated to be $3.3 \mathrm{eV}$ (see Fig. 6). This value is noteworthy both because it corresponds to ultraviolet wavelengths and is thus quite large, but at the same time is significantly lower than the largest calculated gap for this size. This highlights the key role played by both strain and symmetry in determining the optical gap. For clusters of higher symmetry, the molecular orbitals become degenerate, increasing the spacing between energy levels. This can be seen in the density of single-particle states, shown in Fig. 8. The higher symmetry clusters have sharp peaks in the density of states. As the symmetry is broken, the degeneracies are split, leading to a broader, more uniform density of states. Because the optical absorption gaps of high symmetry clusters are enhanced by degeneracy of the molecular orbitals, they are thus more sensitive to perturbation, as evidenced by the large variation in optical absorption gaps between $\mathrm{Si}_{29} \mathrm{H}_{36}$ and the $\mathrm{Si}_{29} \mathrm{H}_{24}$ reconstructions.

\section{CONCLUSIONS}

From high temperature FPMD synthesis calculations, we find that $1 \mathrm{~nm}$ clusters consistently form with noncrystalline, double tetrahedral cores. This result is due to the highly curved underpassivated surface in the early stages of synthesis; it is energetically favorable 
for multiple atoms to move into the interior of the cluster to saturate the maximum number of surface dangling bonds. As the surface is passivated, it freezes around a double tetrahedral core. We find reconstructions unique to highly curved nanostructures which give evidence for larger optical absorption gaps than previously predicted in reconstructed $1 \mathrm{~nm}$ crystalline nanoclusters. The response of the optical absorption gap to the addition of oxygen atoms to the surface of reconstructed crystalline clusters in a single-bonding "bridged" configuration is highly sensitive to the reconstruction. This may provide a unique probe of nanocluster structure, if one can synthesize reconstructed crystalline clusters and measure how the optical absorption gap changes as oxygen is added to the system. In contrast, the gap of noncrystalline clusters is insensitive to the addition of oxygen. These results, in combination with the calculated response of the optical absorption gap to hydrostatic compression, provide evidence that the optical gap of passivated $1 \mathrm{~nm}$ silicon nanoclusters has a lower bound of roughly $3.2 \mathrm{eV}$, which can be significantly increased in clusters with high symmetry and low angular distortion of the silicon bonds away from bulk-like tetrahedral coordination.

\section{ACKNOWLEDGEMENTS}

The authors thank A. Puzder and F. Reboredo for useful discussions. This work was performed under the auspices of the U.S. Department of Energy by University of California Lawrence Livermore National Laboratory under contract No. W-7405-Eng-48. 


\section{REFERENCES}

[1] M. F. Jarrold and J. E. Bower, J. Chem. Phys. 96, 9180 (1992).

[2] E. C. Honea et al., Nature 366, 42 (1993).

[3] U. Röthlisberger, W. Andreoni, and M. Parrinello, Phys. Rev. Lett. 72, 665 (1994).

[4] A. D. Yoffe, Adv. Phys. 50, 1 (2001).

[5] G. Allan, C. Delerue, and M. Lannoo, Phys. Rev. Lett. 78, 3161 (1997).

[6] F. A. Reboredo, A. Franceschetti, and A. Zunger, Appl. Phys. lett. 99, 2972 (1999).

[7] L. Mitas et al., Appl. Phys. Lett. 78, 1918 (2001).

[8] A. Puzder et al. Phys. Rev. Lett. 91, 157405 (2003).

[9] E. W. Draeger et al., Phys. Rev. Lett. 90, 167402 (2003).

[10] D. J. Lockwood et al., Solid State Comm. 89, 587 (1994).

[11] L. T. Canham, Appl. Phys. Lett. 57, 1046 (1990).

[12] T. van Buuren et al., Phys. Rev. Lett. 80, 3803 (1998).

[13] J. P. Wilcoxon et al., Phys. Rev. B 60, 2704 (1999).

[14] L. N. Dinh et al., Phys. Rev. B 59, 15513 (1999).

[15] C.-S. Yang et al., J. Am. Chem. Soc. 121, 5191 (1999).

[16] J. D. Holmes et al., J. Am. Chem. Soc. 123, 3743 (2001).

[17] G. Belomoin et al., Appl. Phys. Lett. 80, 841 (2002).

[18] GP code, version 1.16.0 User's Manual, F. Gygi, LLNL (2003).

[19] D. R. Hamann, Phys. Rev. B 40, 2980 (1989).

[20] P. Giannozzi, private communication. 
[21] CASINO code, version 1.5.4 User's Manual, R. J. Needs et al., University of Cambridge (2002).

[22] A. Williamson et al., Phys. Rev. Lett. 89, 196803 (2002).

[23] L. W. Wang and A. Zunger, J. Phys. Chem. 98, 2158 (1994).

[24] M. Rohlfing and S. G. Louie, Phys. Rev. Lett. 80, 3320 (1998).

[25] I. Vasiliev, S. Ogut and J. R. Chelikowsky, Phys. Rev. Lett. 86, 1813 (2001).

[26] A. Puzder et al., Phys. Rev. Lett. 88, 097401 (2002).

[27] Z. Zhou et al., J. Am. Chem. Soc. 125, 15599 (2003).

[28] L. Patrone et al., J. Appl. Phys. 87, 3829 (2000).

[29] A. Puzder et al., J. Am. Chem. Soc. 125, 2786 (2003).

[30] M. V. Wolkin et al., Phys. Rev. Lett. 82, 197 (1999).

[31] I. Vasiliev, R. M. Martin, and J. R. Chelikowsky, Phys. Rev. B 65, 121302 (2002).

[32] A. Puzder et al., J. Chem. Phys. 117, 6721 (2002). 


\section{TABLES}

TABLE I. Energy gaps for silicon nanoclusters, calculated using both LDA and QMC. For structures created with FPMD, a range of LDA gap energies is given, with QMC gaps calculated only for the structure with the largest LDA gap.

\begin{tabular}{lll}
\hline \hline & $\mathrm{E}_{\text {gap }}^{L D A}(\mathrm{eV})$ & $\mathrm{E}_{\text {gap }}^{Q M C}(\mathrm{eV})$ \\
\hline
\end{tabular}

Unreconstructed

crystalline:

$\begin{array}{lll}\mathrm{Si}_{29} \mathrm{H}_{36} & 3.6^{1} & 5.3(1)^{1}\end{array}$

Reconstructed

crystalline:

\begin{tabular}{lcc}
$\mathrm{Si}_{29} \mathrm{H}_{24}^{\text {nano1 }}$ & 3.1 & $4.5(1)$ \\
$\mathrm{Si}_{29} \mathrm{H}_{24}^{\text {nano2 }}$ & 2.9 & $4.1(1)$ \\
$\mathrm{Si}_{29} \mathrm{H}_{24}^{\text {bulk }}$ & 2.6 & $3.5(1)$ \\
\hline
\end{tabular}

Reconstructed

non-crystalline:

$\begin{array}{lcc}\mathrm{Si}_{29} \mathrm{H}_{22}^{F P M D} & 2.2-2.3 & 3.3(1) \\ \mathrm{Si}_{30} \mathrm{H}_{22}^{F P M D} & 2.2-2.4 & 3.4(1) \\ \mathrm{Si}_{30} \mathrm{H}_{22}^{\text {ideal }} & 2.2 & 3.2(1)\end{array}$

\footnotetext{
${ }^{1}$ Ref. [29]
} 
TABLE II. Energy gaps for silicon nanoclusters with and without oxygen, calculated using both LDA and QMC.

\begin{tabular}{lcc}
\hline \hline & $\mathrm{E}_{\text {gap }}^{L D A}(\mathrm{eV})$ & $\mathrm{E}_{\text {gap }}^{Q M C}(\mathrm{eV})$ \\
\hline $\mathrm{Si}_{29} \mathrm{H}_{36}$ & $3.6^{1}$ & $5.3(1)^{1}$ \\
$\mathrm{Si}_{29} \mathrm{H}_{34} \mathrm{O}^{\text {bridged }}$ & $3.1^{1}$ & $4.7(1)^{1}$ \\
$\mathrm{Si}_{29} \mathrm{H}_{24} \mathrm{O}_{6}^{\text {bulk }}$ & 2.4 & $3.8(1)$ \\
\hline $\mathrm{Si}_{29} \mathrm{H}_{24}^{\text {nano1 }}$ & 3.1 & $4.5(1)$ \\
$\mathrm{Si}_{29} \mathrm{H}_{24} \mathrm{O}^{\text {nano } 1}$ & 2.9 & $4.4(1)$ \\
$\mathrm{Si}_{29} \mathrm{H}_{24} \mathrm{O}_{6}^{\text {nano } 1}$ & 2.3 & $3.8(1)$ \\
\hline $\mathrm{Si}_{29} \mathrm{H}_{24}^{\text {nano2 }}$ & 2.9 & $4.1(1)$ \\
$\mathrm{Si}_{29} \mathrm{H}_{24} \mathrm{O}^{\text {nano } 2}$ & 2.9 & $4.0(1)$ \\
$\mathrm{Si}_{29} \mathrm{H}_{24} \mathrm{O}_{6}^{\text {nano } 2}$ & 2.6 & $4.1(1)$ \\
\hline $\mathrm{Si}_{29} \mathrm{H}_{24}^{\text {bulk }}$ & 2.6 & $3.5(1)$ \\
$\mathrm{Si}_{29} \mathrm{H}_{24} \mathrm{O}^{\text {bulk }}$ & 2.2 & $3.2(1)$ \\
$\mathrm{Si}_{29} \mathrm{H}_{24} \mathrm{O}_{6}^{\text {bulk }}$ & 2.6 & - \\
\hline $\mathrm{Si}_{30} \mathrm{H}_{22}^{\text {ideal }}$ & 2.4 & $3.2(1)$ \\
$\mathrm{Si}_{30} \mathrm{H}_{22} \mathrm{O}^{\text {ideal }}$ & 2.2 & - \\
\hline $\mathrm{Si}_{30} \mathrm{H}_{22}^{F P M D}$ & $2.2(1)$ \\
$\mathrm{Si}_{30} \mathrm{H}_{22} \mathrm{O}^{\text {FPMD }}$ & 2.2 & \\
\hline \hline
\end{tabular}

\footnotetext{
${ }^{1}$ Ref. [29]
} 


\section{FIGURES}
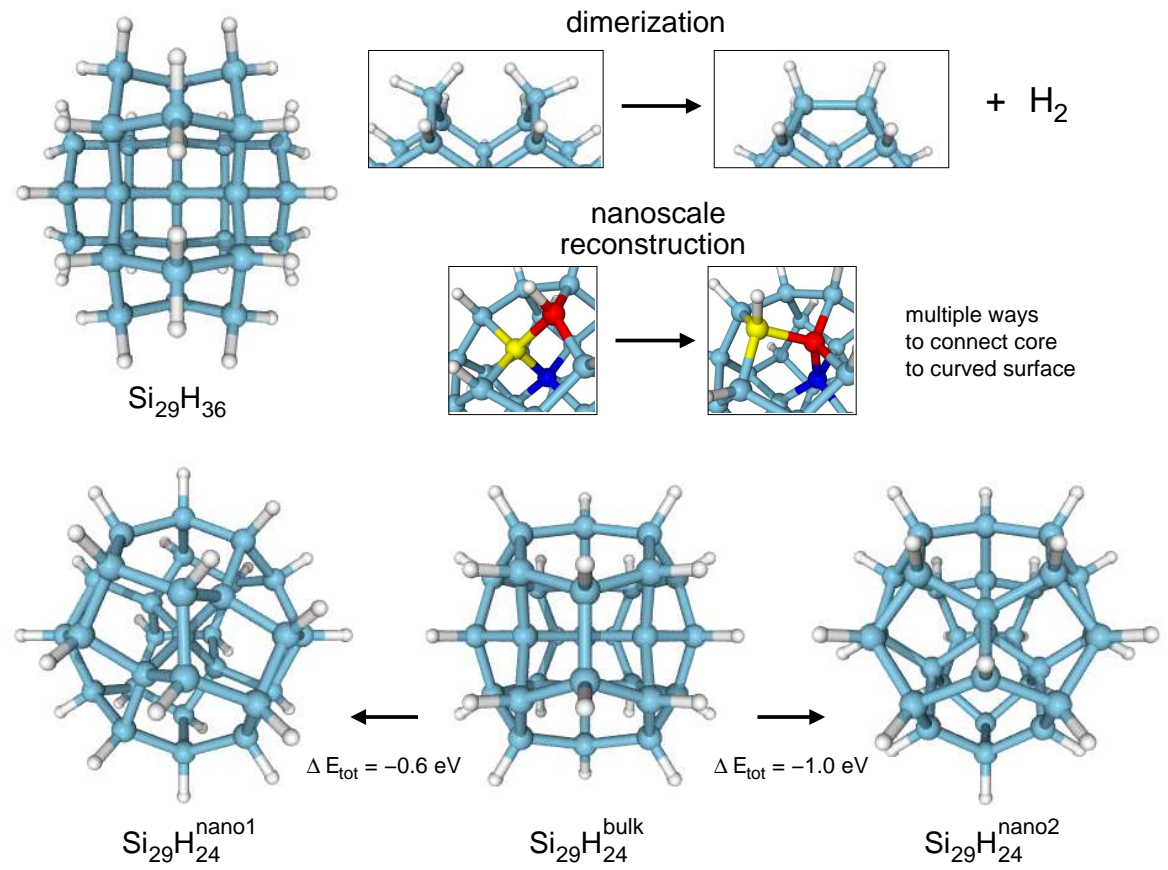

FIG. 1. Different reconstructions of crystalline $\mathrm{Si}_{29}$ : Ideal nanocrystal $\mathrm{Si}_{29} \mathrm{H}_{36}$, equivalent to a spherical piece of bulk silicon with all dangling bonds passivated by hydrogen. Also shown is the $\mathrm{Si}_{29} \mathrm{H}_{24}^{b u l k}$ structure proposed in Ref. [7], based on a bulk-like reconstruction of $\mathrm{Si}_{29} \mathrm{H}_{36}$, along with two additional $\mathrm{Si}_{29} \mathrm{H}_{24}$ reconstructions which are lower in energy than $\mathrm{Si}_{29} \mathrm{H}_{24}^{\text {bulk }}: \mathrm{Si}_{29} \mathrm{H}_{24}^{\text {nano1 }}$ and $\mathrm{Si}_{29} \mathrm{H}_{24}^{\text {nano2 }}$. 


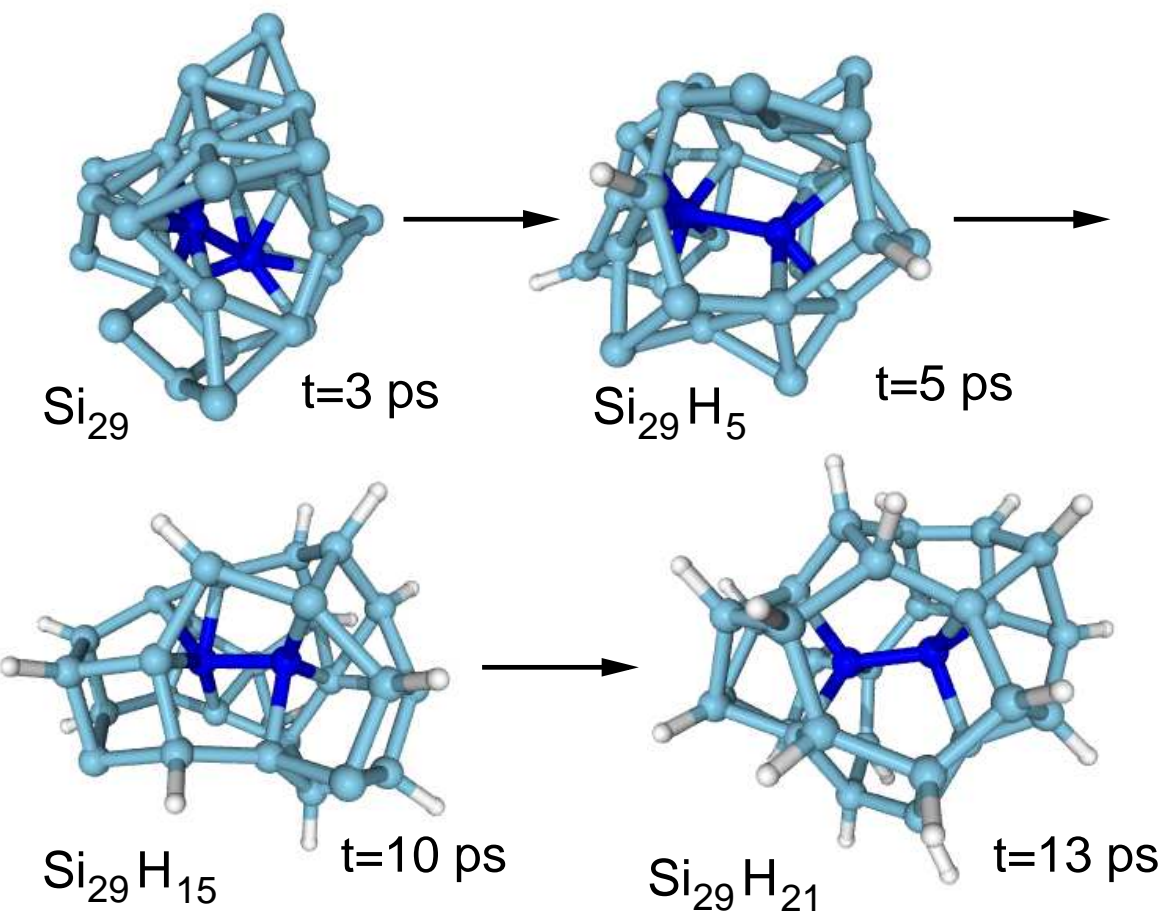

FIG. 2. Snapshots of FPMD simulation of the synthesis of a $1 \mathrm{~nm}$ silicon quantum dot at $\mathrm{T}=1000 \mathrm{~K}$. Interior core atoms are dark blue. 

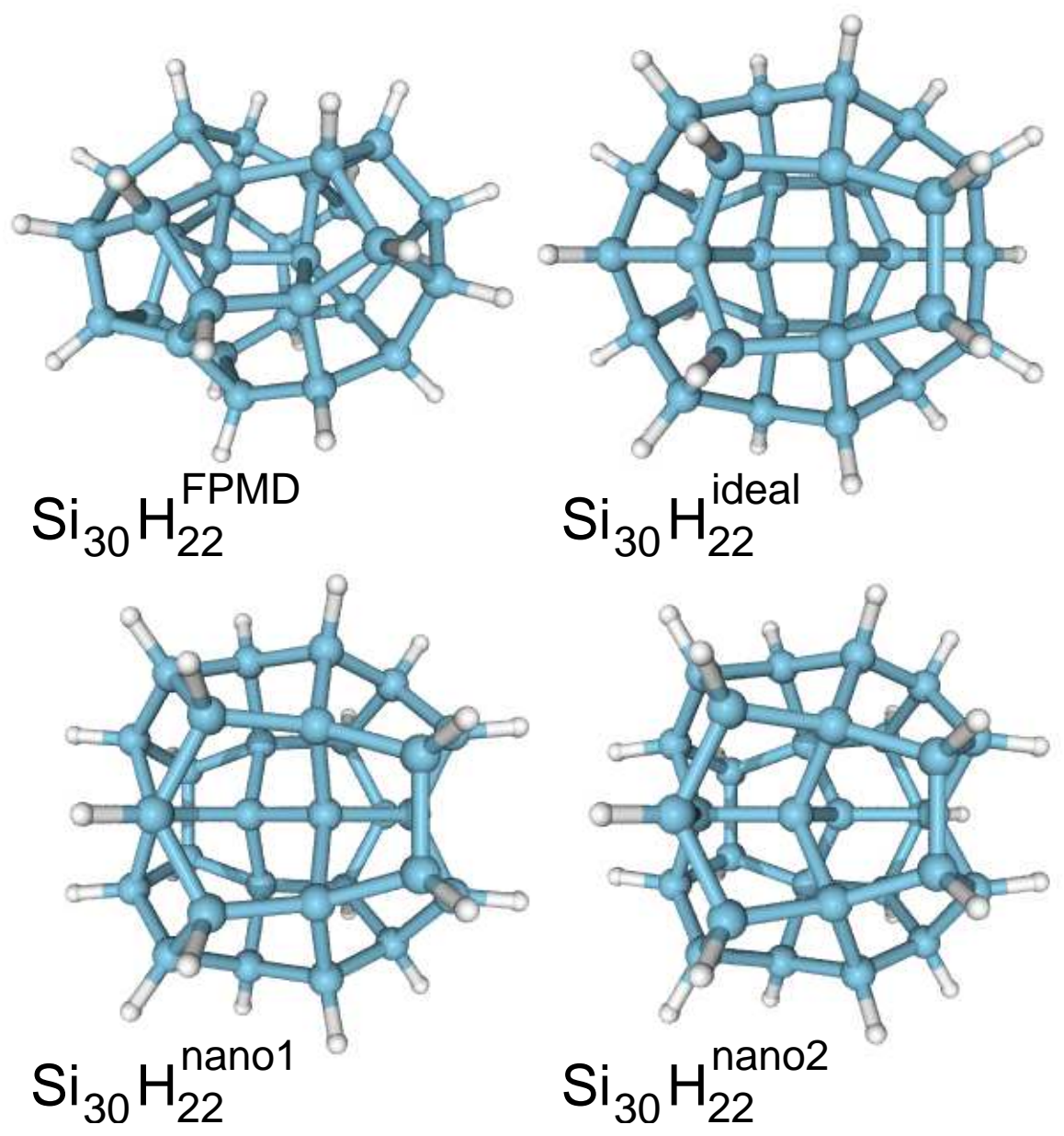

FIG. 3. A representative $\mathrm{Si}_{30} \mathrm{H}_{22}$ structure produced by FPMD, compared with different symmetric reconstructions of $\mathrm{Si}_{30} \mathrm{H}_{22}$. 


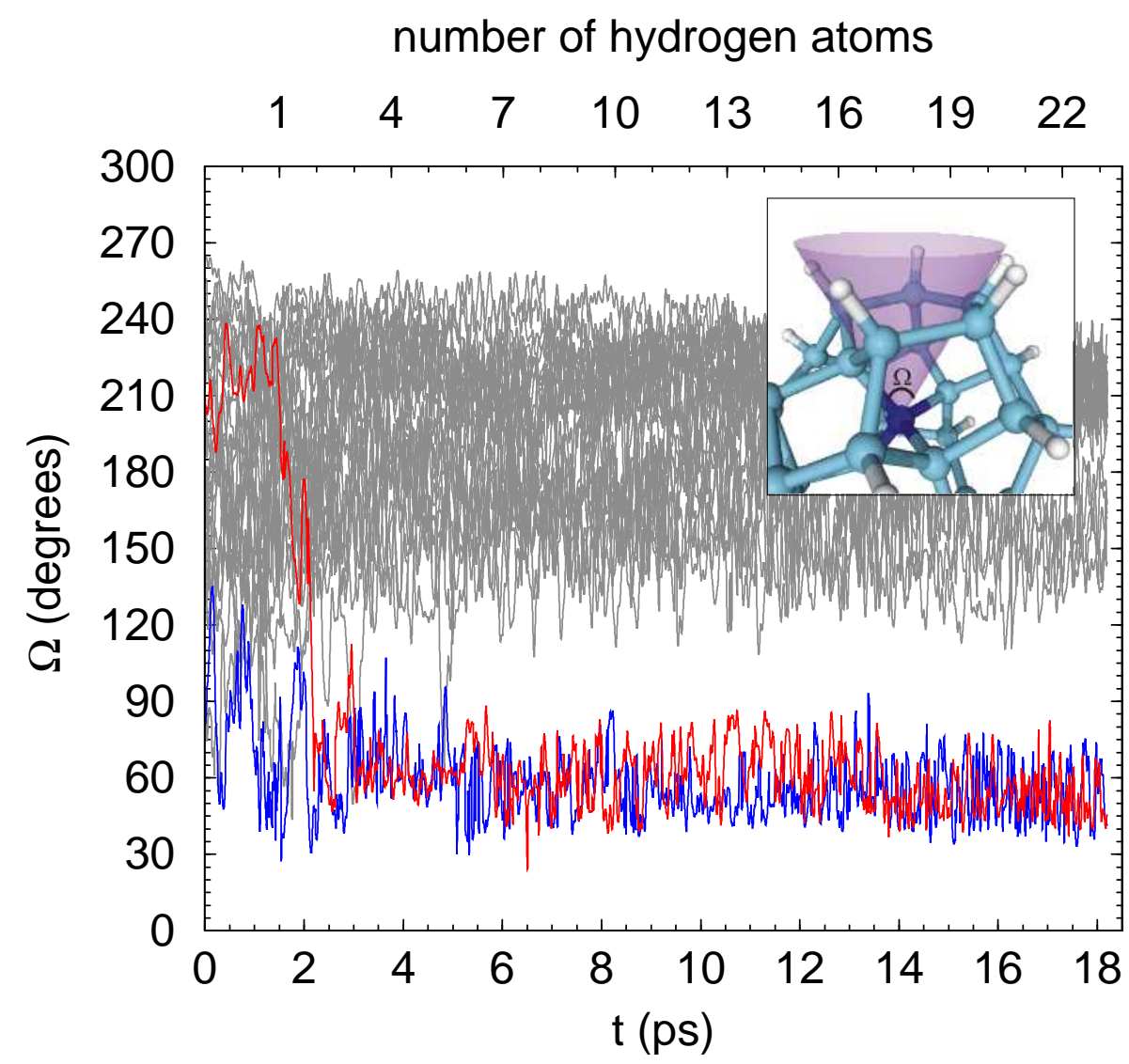

FIG. 4. Maximum solid angle unoccupied by other silicon atoms $\Omega_{i}$ (see text) vs. time in an $\mathrm{Si}_{30} \mathrm{FPMD}$ synthesis run at $\mathrm{T}=1000 \mathrm{~K}$, with a hydrogen atom added to the surface of the cluster every 0.75 ps. The blue and red curves correspond to the two interior core atoms, and the grey curves are surface silicon atoms. The inset shows how $\Omega$ is defined, in this case for an interior atom (dark blue). 


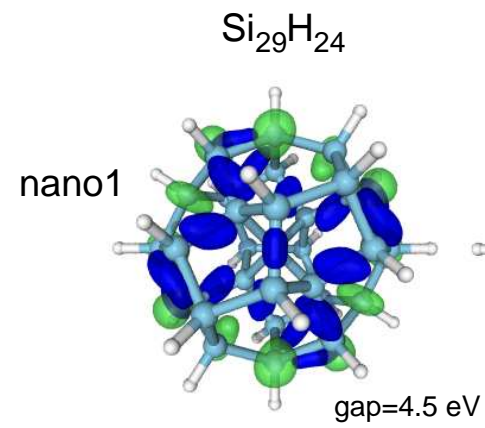

$$
\mathrm{Si}_{29} \mathrm{H}_{24} \mathrm{O} \quad \mathrm{Si}_{29} \mathrm{H}_{24} \mathrm{O}_{6}
$$
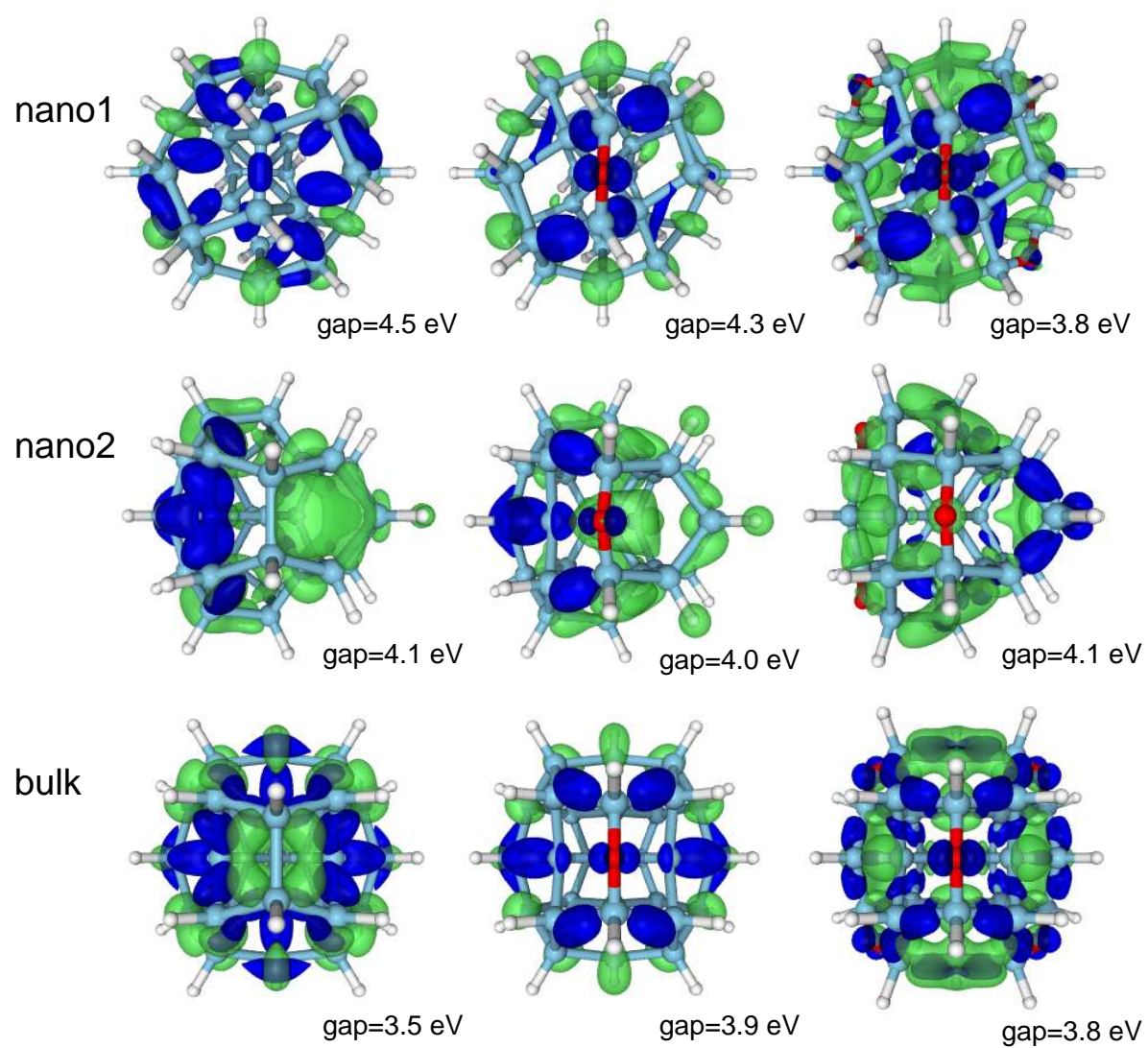

FIG. 5. The effect of oxygen on the HOMO (blue) and LUMO (green) of the three reconstructions of $\mathrm{Si}_{29} \mathrm{H}_{24}$. The isosurface of each normalized state was chosen so that $25 \%$ of the state's total charge density is enclosed. 


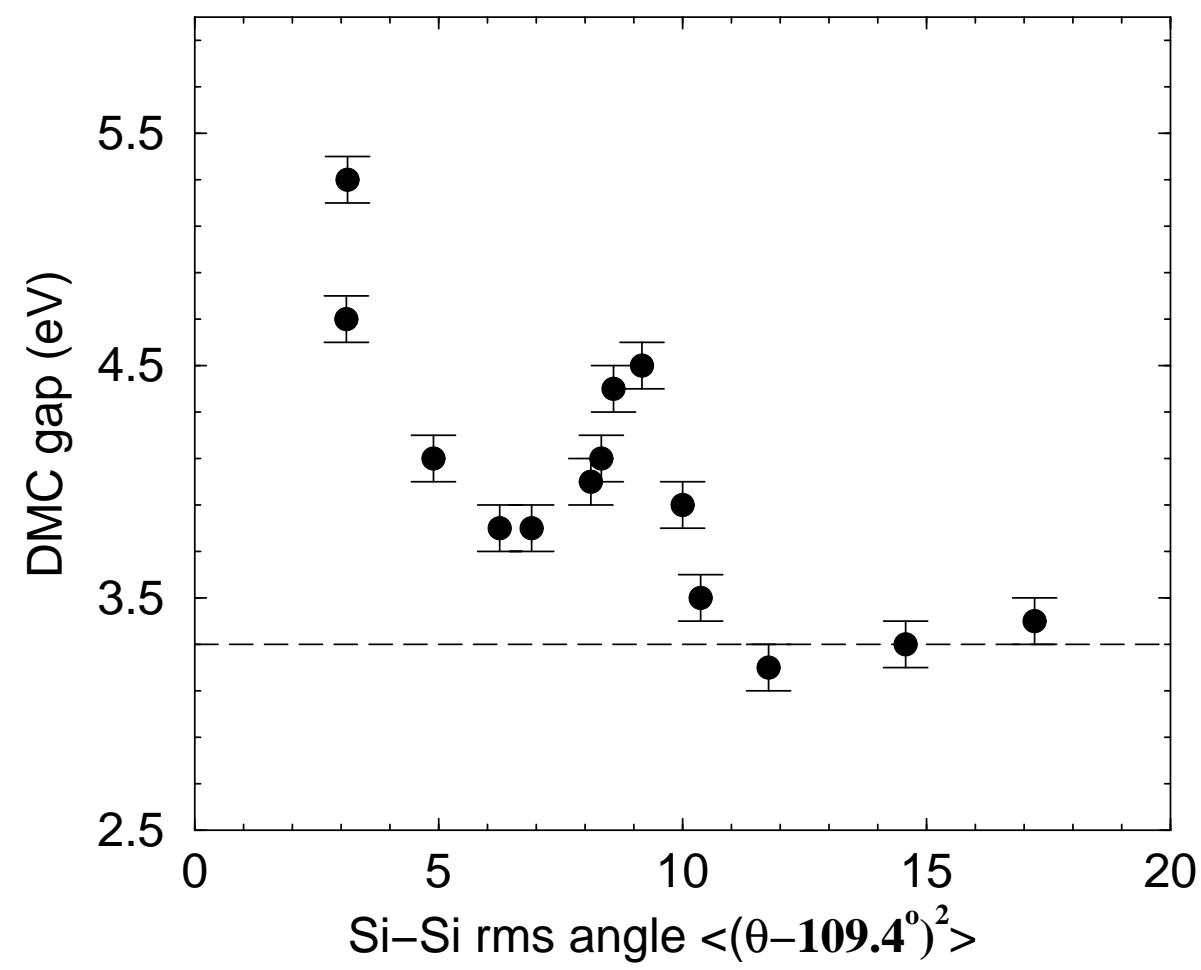

FIG. 6. DMC optical absorption gaps from Tables I and II, plotted vs. angular distortion. The dotted line shows a proposed lower bound on the optical absorption gap.

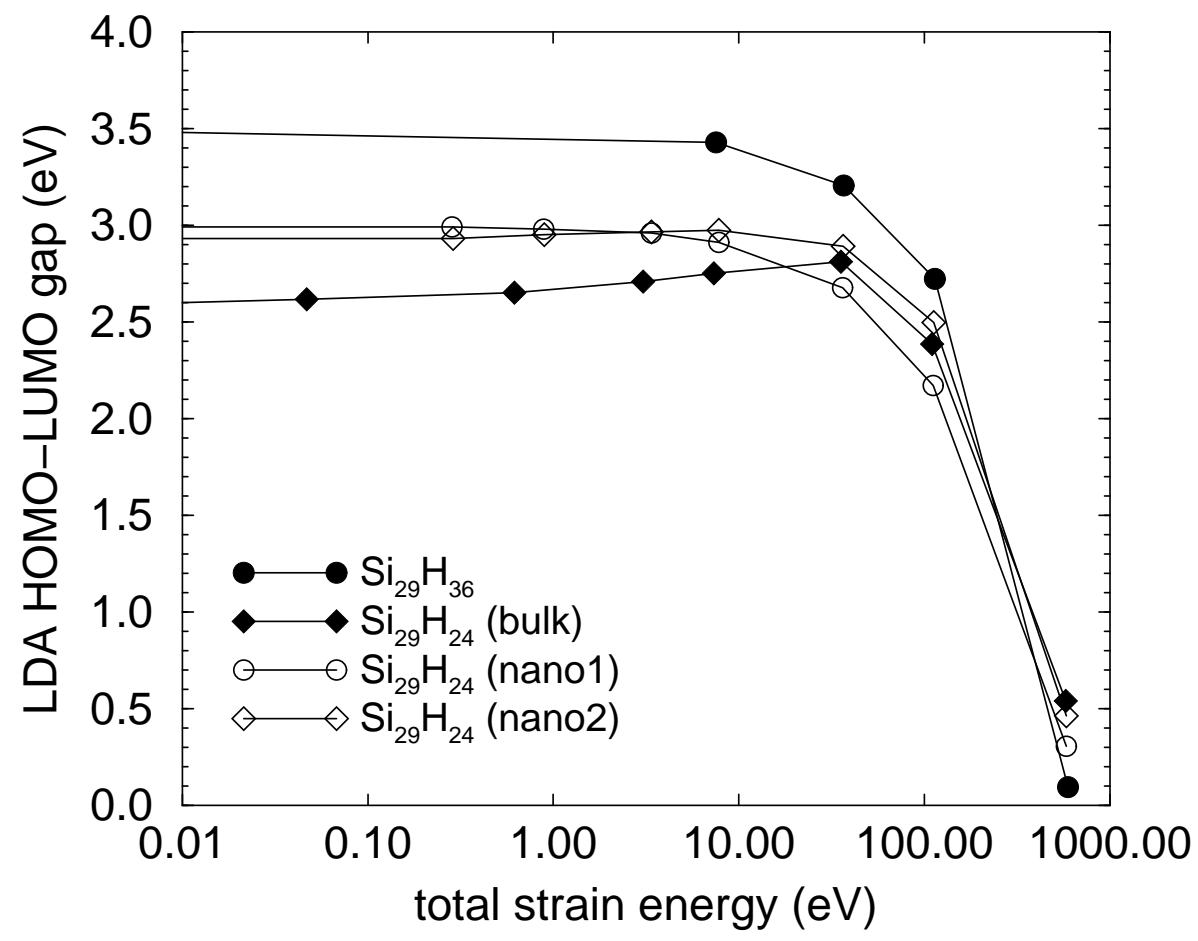


FIG. 7. LDA HOMO-LUMO gap of $\mathrm{Si}_{29} \mathrm{H}_{24}$ reconstructions vs. total energy difference caused by hydrostatic compression.

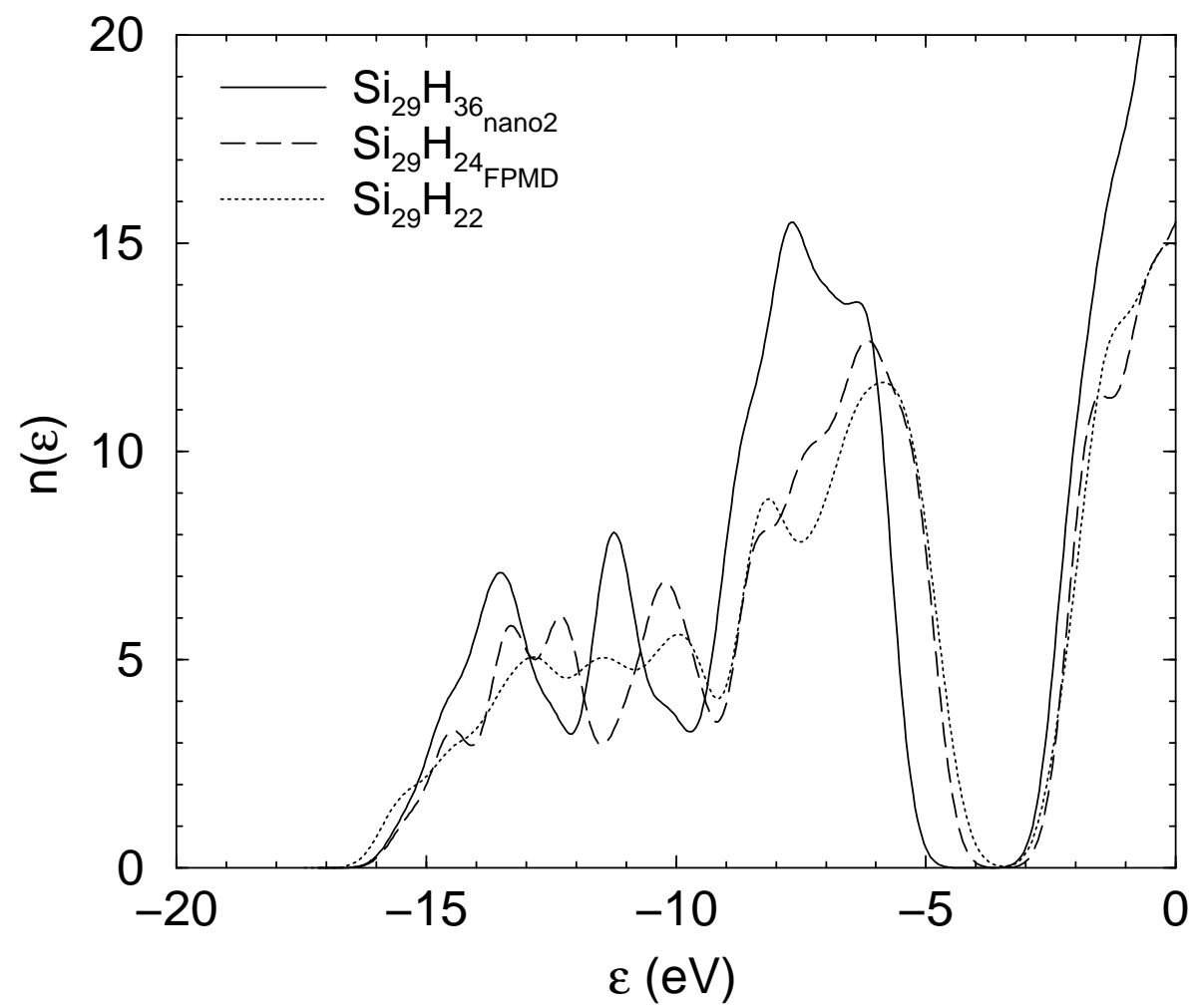

FIG. 8. Density of states for different $\mathrm{Si}_{29}$ nanoclusters, calculated by broadening single-particle LDA eigenvalues with Gaussians of width $0.3 \mathrm{eV}$. For $\mathrm{Si}_{29} \mathrm{H}_{24}$ and $\mathrm{Si}_{29} \mathrm{H}_{22}^{F P M D}$, structures shown are representative of each category. 